\title{
The cost of health care for AIDS patients in Saskatchewan
}

KEVIN P BROWNE, MD, STEVE D SHAFRAN, MD, FRCPC, JOHN M CONLY, MD, CCFP, FRCPC, FACP

ABSTRACT: The medical records of 19 patients with acquired immune deficiency syndrome (AIDS) were reviewed in an attempt to estimate their health care costs. The patients were all male, members of high risk groups and diagnosed between April 1985 and February 1988. Twelve of the patients died; they lived a mean of 240 days (range 0 to 580) after diagnosis, were admitted three times (range one to six) to hospital for 65 total days (range one to 148) for a cost per patient of $\$ 33,721$ (range $\$ 2,768$ to $\$ 64,981$ ) for inpatient care. They made five (range zero to 25) office visits per patient costing $\$ 196$ per patient (range $\$ 0$ to $\$ 4,999$ ) for outpatient care. The seven survivors (one was lost to follow-up) have lived 375 days (range 186 to 551 ) since diagnosis, have been admitted to hospital two times (range zero to seven) for 30 total days (range zero to 86 ) for a total cost per patient of $\$ 14,223$ (range $\$ 0$ to $\$ 39,410$ ) for in- patient care. They have made 11 office/emergency room visits (range zero to 46 ) costing in total $\$ 4322$ (range $\$ 0$ to $\$ 13,605$ ) for outpatient care. The total expenditure was $\$ 546,332$ (\$28,754 per patient), of which total fees to physicians were $\$ 37,210(6.8 \%)$, and estimated costs of laboratory tests $\$ 117,917$ (21.6\%), drugs $\$ 36,930$ $(6.7 \%)$, and medical imaging $\$ 20,794$ (3.8\%). Patients now deceased cost $\$ 416,445$ (mean $\$ 34,704$ per patient), accounting for $76.2 \%$ of overall expenditures. The average medical/surgical and drug costs per patient day in hospital were greater for AIDS patients than for the average medical/surgical patient in the authors' institution. Can J Infect Dis 1990;1(4):127132

Key Words: AIDS, Economic impact, Hospital costs, Inpatient, Outpatient
A LTHOUGH OPINIONS OF THE EXACT RATE OF INcrease vary, it is generally accepted that the number of cases of acquired immune deficiency syndrome (AIDS) in North America will increase in the near future (1). The emergence of greater numbers of AIDS patients will place a growing demand

Department of Medicine, University of Saskatchewan, Saskatoon, Saskatchewan

Presented in abstract form at the V International

Conference on AiDS, Montreal, Quebec, June 1989

Correspondence and reprints: Dr JM Conly. Department of

Medicine/Microbiology. Section of Infectious Diseases,

University Hospital, Saskatoon. Saskatchewan S7N OXO

Received for publication April 25, 1990. Accepted August

13,1990 on the limited resources available within the health care budget. A frequently cited estimate of lifetime health care costs of patients with AIDS in the United States sets the average hospital expenditures of the first 10,000 patients at US\$1.473 billion over their lifetime or US\$147,000 per patient (2). More recent studies of lifetime mean costs have found considerably lower figures than this, ranging from US $\$ 27,571$ to US\$50,380 $(3,4)$. To date, there have been few published reports to determine the health care costs of treating patients with AIDS in Canada $(5,6)$. To determine more detailed and specific data (including mean lifetime costs) on the costs of treating patients with AIDS in Saskatchewan, the authors con- 
ducted a retrospective study of the medical cure costs of patients diagnosed and treated with AIDS between April 1985 and February 1988. In defining the present financial impact of AIDS in the province, it may be possible to identify areas where cost savings could be achieved.

\section{PATIENTS AND METHODS}

Patient selection: Patient data were collected from medical records at University Hospital in Saskatoon and the Plains Health Centre in Regina for 19 of the first 21 patients in Saskatchewan diagnosed with AIDS between April 1985 and February 1988, in accordance with the accepted definition from the Centers for Disease Control in Atlanta (7). These hospitals represent the sites at which the diagnosis was established for all patients and from which follow-up care was coordinated. All patients were well known to their physicians with one-exception (an inmate at a correctional facility with multiple risk factors whose human immunodeficiency virus [HIV] antibody test was reported positive by enzyme-linked immunosorbent assay but not confirmed by Western blot). The complete records of two patients were not available for analysis (both deceased), hence only 19 of the province's first 21 AIDS cases were included in this study.

Data collection: Records were reviewed from the time patients were diagnosed (usually during a hospital admission) to the time of their death or February 1988. Outpatient records were obtained from the office charts of the department of medicine, Plains Health Centre and from the section of infectious diseases, department of medicine, University Hospital. Efforts were made to ensure that all outpatient visits for which records were available were included, but it is acknowledged that occasional outpatient visits and outpatient laboratory services may have been missed. Hospital use outside of the major centres was included; these visits were both few and well documented.

In this paper the terms 'costs' and 'charges' are used interchangeably. The data collected refer to charges obtained from the Medical Care Insurance Commission billing codes, the University Hospital pharmacy rates, and the University Hospital administration (financial services) for hospital per diem funding rates. The charges for physicians' services would be identical to costs, since extra billing outside the provincial health care insurance plan does not occur; medication charges and costs would also be similar. However, since it is frequently impossible to obtain the actual costs of a laboratory or medical imaging examination on a per unit basis, while the Medical Care Insurance
Commission charges were readily available, this represented an area where charges and costs may not have been identical. However, the differences were small and were not expected to influence the overall results to any significant degree.

\section{ASSESSMENT OF COSTS}

Fees to physicians and surgeons: The physician visits, consultations and referrals documented with reports were tabulated by specialty and the procedures performed cross-referenced for later consideration. Repeat visits were recorded and the costs assessed according to the Medical Care Insurance Commission billing codes for October 1, 1986, which represented the rates in effect during the time course of the study and up to October 1, 1988 (8). Since Saskatchewan pathologists are employed on a salaried basis and do not bill the Medical Care Insurance Commission for the majority of their services, the value of their services was determined using the Medical Care Insurance Commission codes appropriate for out-of-province patients or for services charged against a research grant. These fees were applied whenever a pathologist's report was offered in reference to biopsy and/or body fluid specimens. Procedures compensated by the Medical Care Insurance Commission were recorded for inpatient versus outpatient visits with costs assigned based on October 1, 1986 rates. The records were cross-checked with pathologists' reports and summary letters for accuracy.

Inpatient care by the attending physician was assessed according to rates specified under the 1986 billing fees based on the number of inpatient days, assuming care on a medical ward by a specialist in internal medicine. Figures are in Canadian dollars with the exception of quoted US costs, which are in US dollars.

Hospital and office costs: Overall hospital costs per patient day for medical wards at University Hospital and Plains Health Centre were based on the University Hospital per diem funding rate in 1986. It was not possible to complete a specific cost per bed per patient per day for the intensive care unit beds, but figures for staffing costs in the intensive care unit in 1986 were made available and added to the basic per diem rate minus the average staff charges per bed per day for a medi$\mathrm{cal} /$ surgical bed. It was expected this would better approximate the increased expenses inherent in operating intensive care unit beds, and is in keeping with the approach used in previous studies $(3,4)$.

The per diem rate of funding normally includes the costs of medical imaging, laboratory tests, medication and other investigations. However, it 
TABLE 1

Total cost for medical care for 19 acquired immune deficiency syndrome patients in Saskatchewan

\begin{tabular}{lrc}
\hline & Cost & Percentage \\
\hline Inpatients & & \\
Hospital & $\$ 472,485$ & 86.5 \\
Physician & $\$ 31,729$ & 5.8 \\
Outpatients & & \\
Physician & $\$ 5,481$ & 1.0 \\
Drugs and tests & $\$ 36,637$ & 6.7 \\
Total & $\$ 546,332$ & 100 \\
\hline
\end{tabular}

was possible that these costs would be higher for AIDS patients compared to other medical patients if more of these services were required, so each category was tracked and tabulated separately for later comparison to totals. It should be emphasized that these costs would be otherwise buried because of global budget funding, and that the only way to appreciate them would be to extract them from the charts and apply the best available cost estimates. For the sake of simplicity the actual per diem funding rate was still used in the calculation of total costs without making allowances for the greater use of hospital services by AIDS patients. The separate tracking of these costs was done simply to provide a comparison with other medical patients for similar services. Within a given fiscal year, with a fixed global budget for such services, no additional costs are actually appreciated. It is recognized, however, that the final cost for hospital services used by AIDS patients may be underestimated.

The costs for laboratory work-up were determined from the 1986 Medical Care Insurance Commission billing codes for laboratory medicine. These codes assume that the work is done at a laboratory supervised by a pathologist. Although it was possible to obtain the actual cost estimates for tests performed at University Hospital for 1988, the same data were not available for 1986; therefore, the Medical Care Insurance Commission values for 1986 were used for consistency. It was the opinion of the laboratory supervisory staff that the 1986 figures were conservative but representative estimates for these costs.

The medical imaging was performed at the respective hospital's department of radiology and was funded within the hospital budget. The costs of the services were assessed according to the Medical Care Insurance Commission 1986 billing fees (ie, as though the services were performed at a private clinic), including costs for computed tomography scans.

Medication costs were estimated by tabulating the actual number of drug/dose combinations from patient charts and outpatient reports, and assessing costs based on the 1986 University Hospital pharmacy rates. These costs are lower than those of private pharmacies and so are conservative estimates. In general inpatient data are more reliable than outpatient data because they may be cross-checked from several sources. Medication costs included the costs of azidothymidine, which was used in six patients.

It was not possible to obtain reliable data concerning employment history from the charts, and interviews with patients or their families were not available, so no attempt has been made to estimate this indirect cost.

Analysis: The data are presented using both median and mean values where applicable to allow comparisons with other studies. It should also be noted that the lifetime costs (total of all costs from diagnosis to death) incurred by the 12 deceased patients and the costs of the surviving seven patients until February 1988 were lumped together to achieve total costs to the province up to this point. Unless otherwise specified, lifetime costs refer only to the deceased patients.

\section{RESULTS}

Clinical characteristics: The patients were all males and had a median age of 30 years (range 22 to 63). Risk group categorization was as follows: homosexual/bisexual (14 patients), blood/blood product recipient (three), intravenous drug user (one), and homosexual/bisexual plus intravenous drug user (one). Twelve of the 19 patients were deceased. Eighteen of the patients presented with AIDS and one had been previously followed for a brief period prior to diagnosis. Eleven patients had later opportunistic infections or HIV dementia; five died after their initial presentation without another opportunistic infection; and three developed no further opportunistic infections until the time of analysis. Six patients received treatment with azidothymidine. Four patients received secondary prophylaxis for Pneumocystis carinii pneumonia.

The median survival time after diagnosis was 268 days (range zero to 580 ) for deceased patients and 361 days (range 186 to 551) for patients still alive. The mean survival time after diagnosis was 240 days. One of the deceased patients died about $12 \mathrm{~h}$ after admission to the intensive care unit with a diagnosis of pneumocystosis. Because of the substantial costs incurred with this brief hospitalization, this patient was included in the study. The 12 deceased patients were admitted to hospital a mean of three times (range one to six) for 65 total days (range one to 148) for a total cost per patient of $\$ 33,721$ (range $\$ 2,768$ to $\$ 64,981$ ) 
TABLE 2

Total expenditures for Saskatchewan acquired immune deficiency syndrome patients for drugs, laboratory, medical imaging, physicians' fees, and per diem hospital funding for inpatients versus outpatients, alive versus dead

\begin{tabular}{|c|c|c|c|c|c|}
\hline \multirow[b]{2}{*}{ Category } & \multicolumn{2}{|c|}{ Outpatient } & \multicolumn{2}{|c|}{ Inpatient } & \multirow[b]{2}{*}{ Total } \\
\hline & Alive & Dead & Alive & Dead & \\
\hline Medications & $\$ 14,211$ & $\$ 3,017$ & $\$ 6,413$ & $\$ 13,289$ & $\$ 36,930$ \\
\hline Laboratory & $\$ 8,137$ & $\$ 4,750$ & $\$ 20,741$ & $\$ 84,289$ & $\$ 117,917$ \\
\hline Medical imaging & $\$ 4,847$ & $\$ 1,675$ & $\$ 3,007$ & $\$ 11,265$ & $\$ 20,794$ \\
\hline Per diem hospital rate* & - & - & $\$ 91,606$ & $\$ 380,879$ & $\$ 472,485$ \\
\hline \multicolumn{6}{|l|}{ Physician's fees } \\
\hline Procedures & $\$ 1,138$ & $\$ 417$ & $\$ 4,459$ & $\$ 11,184$ & $\$ 17,198$ \\
\hline Total & $\$ 30,327$ & $\$ 11,791$ & $\$ 99,560$ & $\$ 404,654$ & $\$ 546,332$ \\
\hline Percentage of grand total & 5.6 & 2.2 & 18.2 & 74.1 & \\
\hline
\end{tabular}

-The per diem hospital rate includes the costs of medications, laboratory tests and medical imaging, which are presented for comparative purposes only

\section{TABLE 3}

Comparison of mean life time costs, mean number of lifetime admissions, mean number of hospital days per lifetime, mean length of stay, and mean survival time after diagnosis for acquired immune deficiency syndrome patients from selected studies

\begin{tabular}{lccccc}
\hline Location (reference) & Life time costs & $\begin{array}{c}\text { Number of } \\
\text { admissions }\end{array}$ & $\begin{array}{c}\text { Hospital days } \\
\text { Length of stay }\end{array}$ & $\begin{array}{c}\text { Survival time after } \\
\text { diagnosis }\end{array}$ \\
\hline Saskatchewan & $\$ 34,704$ & 3.2 & 3.6 & 65 & 19 days \\
Massachusetts (4) & $\$ 50,380$ & 3.2 & 34.7 & 21 days & 395 days \\
San Francisco (3) & $\$ 27,571$ & 4.8 & 52.2 & 11.7 days & 224 days \\
San Francisco (3) (adjusted) & $\$ 41,499$ & NA & 168 & NA days & 341 days \\
United States (2) & $\$ 147,000$ & & & 392 days \\
\hline
\end{tabular}

NA Not available

for inpatient care and made five office visits (range zero to 25) per patient costing $\$ 196$ (range $\$ 0$ to $\$ 4,999$ ) per patient for outpatient care. The seven survivors (one was lost to follow-up) have been admitted two times (zero to seven) to hospital for 30 total days (range zero to 86 ) for a total cost per patient of $\$ 14,223$ (range $\$ 0$ to $\$ 39,410$ ) for inpatient care and have made 11 office/emergency room visits (range zero to 46) costing a total of $\$ 4322$ (range $\$ 0$ to $\$ 13,605$ ) for outpatient care. Median values for number of admissions, total hospital days, and number of visits were not significantly different from mean values and are not presented.

The total medical care expenditures for 19 AIDS patients in Saskatchewan to February 1988 are presented in Table 1. The data illustrate separate total hospital costs, inpatient and outpatient physician costs and outpatient laboratory and drug costs. Inhospital costs accounted for $86.5 \%$ of total care costs. A cost breakdown of Saskatchewan expenditures in more detail (Table 2) shows how funds have been used for medications, laboratory tests and radiological procedures. Under global budget funding these items are normally included in 'per diem hospital funding' but were separately tracked and are thus presented so that expenditures in these areas may be compared. The overall expenditures reveal that a total of $\$ 546,332$ has been spent on 19 AIDs patients, or $\$ 28,754$ per patient whether alive or dead. Physicians' professional costs accounted for only $5.8 \%$ of the total. Drug costs were similar to those for professional services $(6.7 \%)$, medical imaging about one-half that much (3.8\%), and laboratory investigations about one-fifth of the total (21.6\%).

Reclassifying the costs based on whether patients were alive or dead and whether expenditures were for outpatient or inpatient services indicated that patients who were dead accounted for greater total and mean per patient costs in all areas except drugs $181 \%$ of total hospital costs, $44 \%$ of drug costs, $75 \%$ of laboratory costs, $62 \%$ of medical imaging costs, and $70 \%$ of physicians' professional costs). The costs for inpatient care likewise accounted for greater costs in all areas (53\% of drug costs, $89 \%$ of laboratory costs, $69 \%$ of medical imaging costs, and 85\% of physicians' professional fees).

The average cost per patient per day in 1986 for medications and for medical/surgical costs for University Hospital were compared to the same data for AIDs patients during the same time period.

Medical/surgical costs were defined as the per 
diem mean daily costs of the medical/surgical patient less staff, lodging and drug costs. This cost thus included the costs of laboratory tests, medical imaging, ancillary services and disposable items. The mean daily drug costs for the average medi$\mathrm{cal} /$ surgical patient and AIDS patients were $\$ 12.77$ and $\$ 19.94$, respectively. The mean daily costs for the medical/surgical patient versus the AIDS patient was $\$ 32.32$ and $\$ 120.75$, respectively.

The mean total cost per admission by presenting diagnosis was also determined. $P$ carinii pneumonia, which was the most frequent presenting diagnosis had a mean cost per admission of $\$ 8,660$, and Kaposi's sarcoma $\$ 7,565$ per admission. Other opportunistic infections as a presenting diagnosis were associated with the highest mean cost of $\$ 18,315$.

A comparison of mean lifetime charges, number of admissions, hospital days, length of stay and survival time after diagnosis with comparison to other major published studies is depicted in Table 3.

\section{DISCUSSION}

The mean survival time of 240 days is comparable to other studies which have found mean survival times between 224 and 392 days (2-4). The median survival time of 268 days for the present is comparable to a recent Manitoba study which found a median survival of 261 days (5). The present data indicate that the majority of costs associated with AIDS accrue from hospitalization. Not unexpectedly, patients who have already died have greater health care costs associated with them. Overall $74 \%$ of the total health care expenditures for AIDS patients are associated with inpatient care for those who have died from the disease. The mean lifetime charges for the present patients are comparable to those from United States centres $(1,3,4)$ and from a Manitoba study (5), all of which were conducted within the same time frame.

The authors were able to determine the total cost per admission depending on admitting diagnosis but not the lifetime costs depending on the diseases; these can be compared to other available data $(3,4)$. In San Francisco, $P$ carinii pneumonia had the most expensive cost per admission $(\$ 14,120)$ and the longest mean length of stay (18.1 days) (3). In Massachusetts the cost per admission of $P$ carinii pneumonia ranked third but its lifetime cost second $(\$ 13,520$ and $\$ 54,576$, respectively) with 'other infections' being the most costly (4). Kaposi's sarcoma lifetime cost $(\$ 32,386)$ was less. In the present study P carinii pneumonia ranked as the second most costly disease per admission behind 'others' $\mathbf{( \$ 8 , 6 6 8}$ versus
$\$ 18,315)$ and was more expensive than Kaposi's sarcoma $(\$ 7,650)$. Patients with Kaposi's sarcoma had other opportunistic infections which may have inflated the cost per admission. The present data are similar to those of Fanning et al (6) who found, in a Toronto hospital, the costs per admission for pneumocystosis and Kaposi's sarcoma were $\$ 11,822$ and $\$ 12,020$, respectively.

Scitovsky et al (1) estimated the total direct personal medical costs for AIDS patients in the United States to be $\$ 630$ million in 1985 , which will increase to $\$ 8,544$ million by 1991 . They estimated the total direct personal costs to comprise about $13 \%$ of estimates of total costs, so that the total direct and indirect costs by 1991 will amount to almost $\$ 66.5$ billion. In Canada the estimates provided by the Federal Centre for AIDS project a total number of 5500 AIDS patients by 1991 , based on the average of logistic and polynomial models (9). Using the per patient cost figures for Saskatchewan and excluding inflationary increases, this projection would represent \$147 million total direct costs up to that year. With an increase of $12 \%$ in physician's fees and a $13 \%$ increase in medication and the per diem hospital funding rate since 1986 in Saskatchewan, this total figure would be correspondingly increased. This presupposes the costs in Saskatchewan to be representative of those for the rest of Canada. Estimates for direct personal costs of AIDS patients in Canada in $1987-88$ were $\$ 82,500$ per person per year, with hospital costs accounting for twothirds of this total (10). Extrapolation of the present data, which were based on a detailed analysis, provides a direct personal cost of $\$ 52,750$ per annum which compares favorably with the overall Canadian estimate.

It is recognized that estimates of future costs based on these data are tenuous and may not be valid if significant changes occur in present patterns of practice and expenditure. Many factors may affect the provision of care and dramatically influence future expenditures. The widespread availability of azidothymidine and other antiretroviral compounds will add some direct costs but may have major cost reducing effects by decreasing the number of opportunistic infections and thus reducing costly hospitalizations. Similarly, earlier diagnosis and intervention, refinements in diagnostic methods and alternatives to acute hospital care may also favorably influence costs. Whether or not the authors' experience with health care costs of these 19 patients is representative of current costs is an assumption which can only be supported when future data become available. Nonetheless the data for these patients was methodically and 
precisely collected and the authors believe it represents an accurate assessment of the costs associated with their care during the designated time period.

Recommendations made by several authors $(3-5,11)$ may apply to Saskatchewan. It is clear that the majority of health care costs accrue from inpatient care for patients who are in the final months of life.

Present data provide support for this observation and emphasize the need for alternatives to hospital care. The authors suggest that improved community support services for AIDS patients will enable more patients to be discharged from hospital early into a supervised but less costly environment. There is anecdotal evidence to suggest that many AIDS patients would prefer to spend their remaining time in the company of their friends and/or family where possible as an alternative to a conventional hospital stay, although there are no published surveys as yet to substantiate this. Alternatives to hospitalization which deserve emphasis in Canada include home care, hospice care and halfway houses. These methods would serve to decrease the major burden of care, which tends to fall on the large hospitals in major urban centres. These alternatives would provide a less expensive option to inpatient care when the individual requires care at a level less than that required in an acute care hospital. It is estimated

\section{REFERENCES}

1. Scitovsky AA, Rice DP. Estimates of the direct and indirect costs of the acquired immunodeficiency syndrome in the United States, 1985, 1986, 1991. Public Health Rep 1987;102:5-17.

2. Hardy AM, Rauch K, Echenberg D, et al. The economic impact of the first 10,000 cases of acquired immunodeficiency syndrome in the United States. JAMA 1986;255:209-15.

3. Scitovsky AA, Cline M, Lee PR. Medical care costs of patients with AIDS in San Francisco. JAMA 1986;256:3103-6.

4. Seage GR, Landers JD, Barry A, Groopman J, Lamb GA, Epstein AM. Medical cure costs of AIDS in Massachusetts. JAMA 1986;256:3107-9.

5. Aoki FY, Bow EJ, Thomson MJ. Costs of medical care for Manitoba's first 17 AIDS patients. Clin Invest Med 1987;510:B90. (Abst)

6. Fanning M, Harmon T, Sheperd FA, Velland H, Minnick S. Influence of disease and casemix severity on the hospital costs of caring for AIDS patients. III. International Conference on AIDS, that the cost of home care would be substantially less than daily hospital care. Hospice care is now available in Canada, and the first free-standing facility, Casey House, opened its doors in Toronto in 1988. Halfway houses could be used as temporary accommodation for individuals requiring neither home care nor hospice care.

Other modalities to achieve cost reduction in the care of AIDS patients include the refinement of diagnostic techniques for opportunistic infections, a reduction in the number of diagnostic tests as physicians gain experience with this group of patients, increased use of outpatient clinics for diagnosis, treatment and continuing care, preventive treatments (primary and secondary prophylaxis for $P$ carinii pneumonia) and the development of specialized health care teams within the hospital sector to maximize efficiency and reduce the need for training and educating new staff.

The costs of treating people with HIV infection and AIDS are significant and will continue to have a major impact on Canada's health care system. The data presented in this study do not address the equally significant nonpersonal direct costs (screening, counselling, education) and the potentially large indirect costs to society through lost individual productive capacity. Nonetheless, recognition of direct personal costs allows a framework to be built from which strategies to reduce costs may be developed.

Washington, DC, 1987:45.

7. CDC. Revision of the CDC surveillance case definition for acquired immunodeficiency syndrome. MMWR 1987;36(Suppl 1S): 1S-14S.

8. Saskatchewan Medical Care Insurance Commission Payment Schedule. Regina: Saskatchewan Health, October 1, 1986.

9. Bureau of Epidemiology and Surveillance. Surveillance update: AIDS in Canada. Federal Centre for AIDS. Ottawa: Health and Welfare Canada, February 1, 1988, Report 2.0:2.

10. Royal Society of Canada. Costs of the AIDS epidemic. In: AIDS: A Perspective for Canadians. Summary Report and Recommendations. Montreal' l'Imprimerie Cooperative Harpell, 1988:8-10.

11. Coolfont Report: A PHS plan for prevention and control of AIDS and the AIDS virus. Public Health Rep 1986;101:341-8.

12. Andrulis DP, Beers VP, Bently JD, Gage LS. The provision and financing of medical care for AIDS patients in US public and private teaching hospitals. JAMA 1987;258:1343-6. 


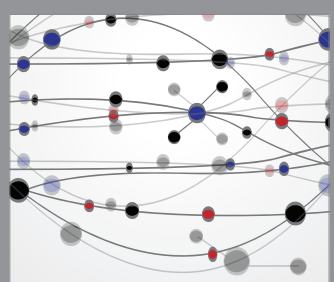

The Scientific World Journal
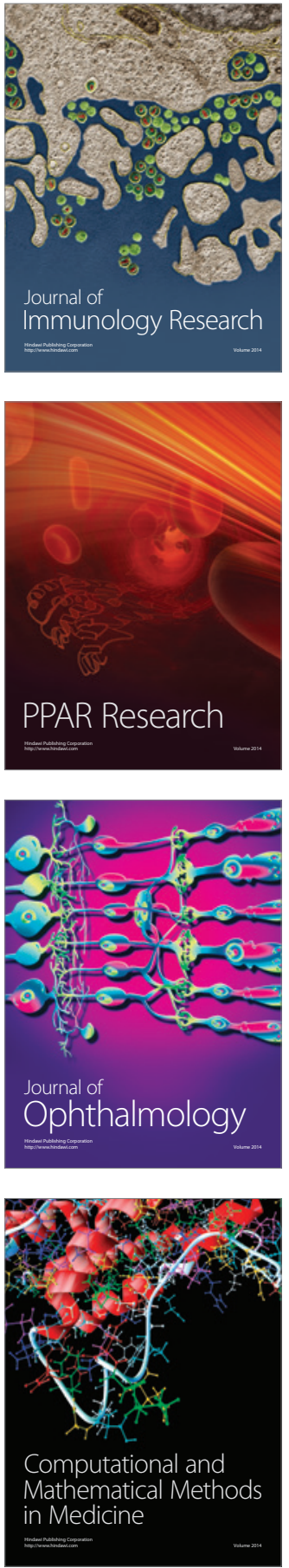

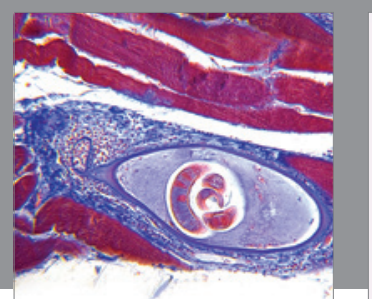

Gastroenterology Research and Practice

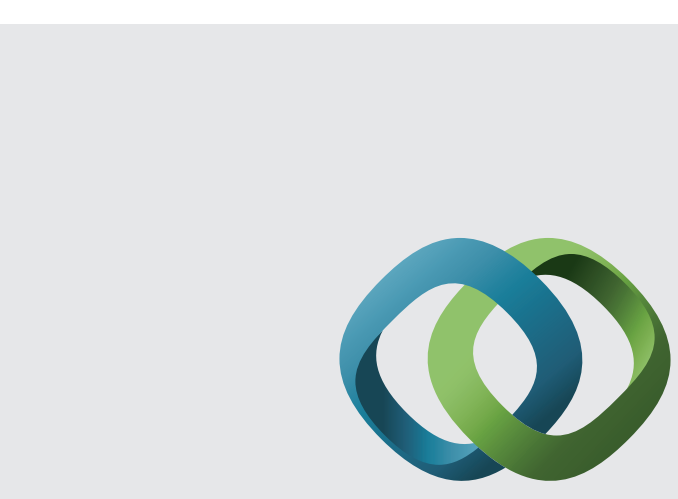

\section{Hindawi}

Submit your manuscripts at

http://www.hindawi.com
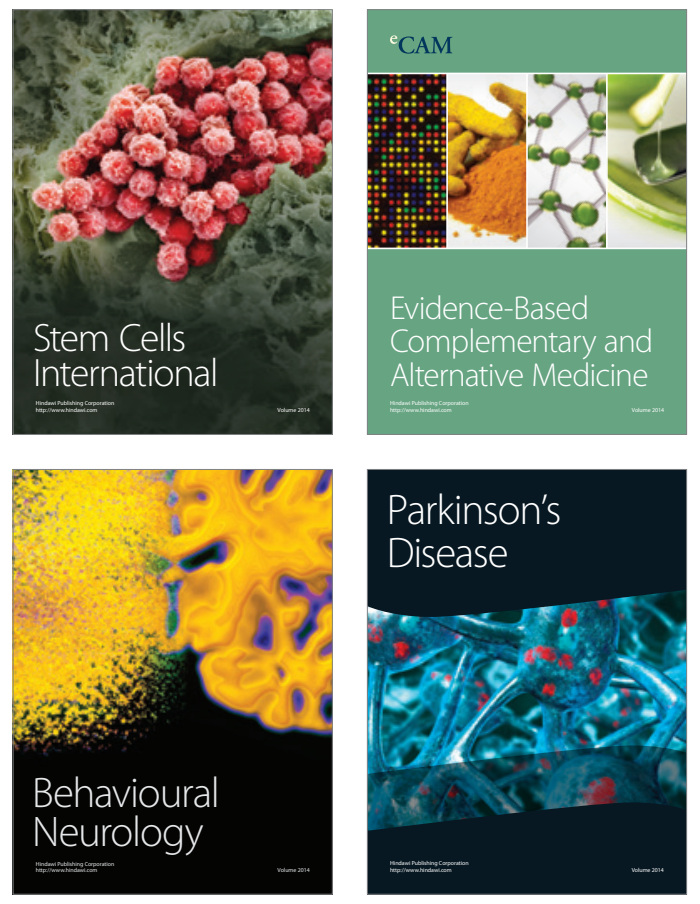
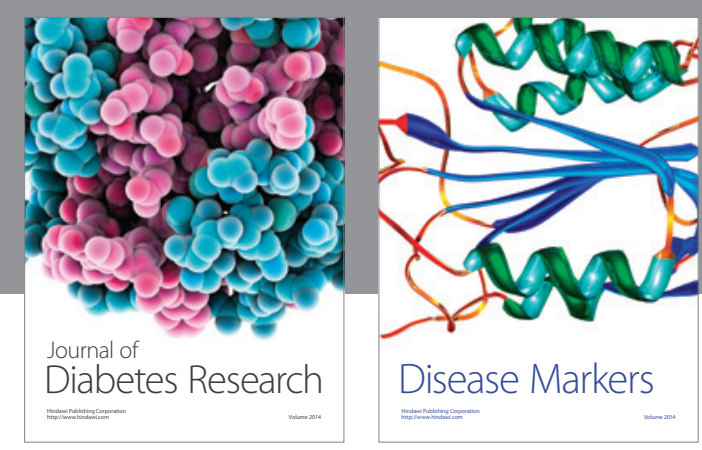

Disease Markers
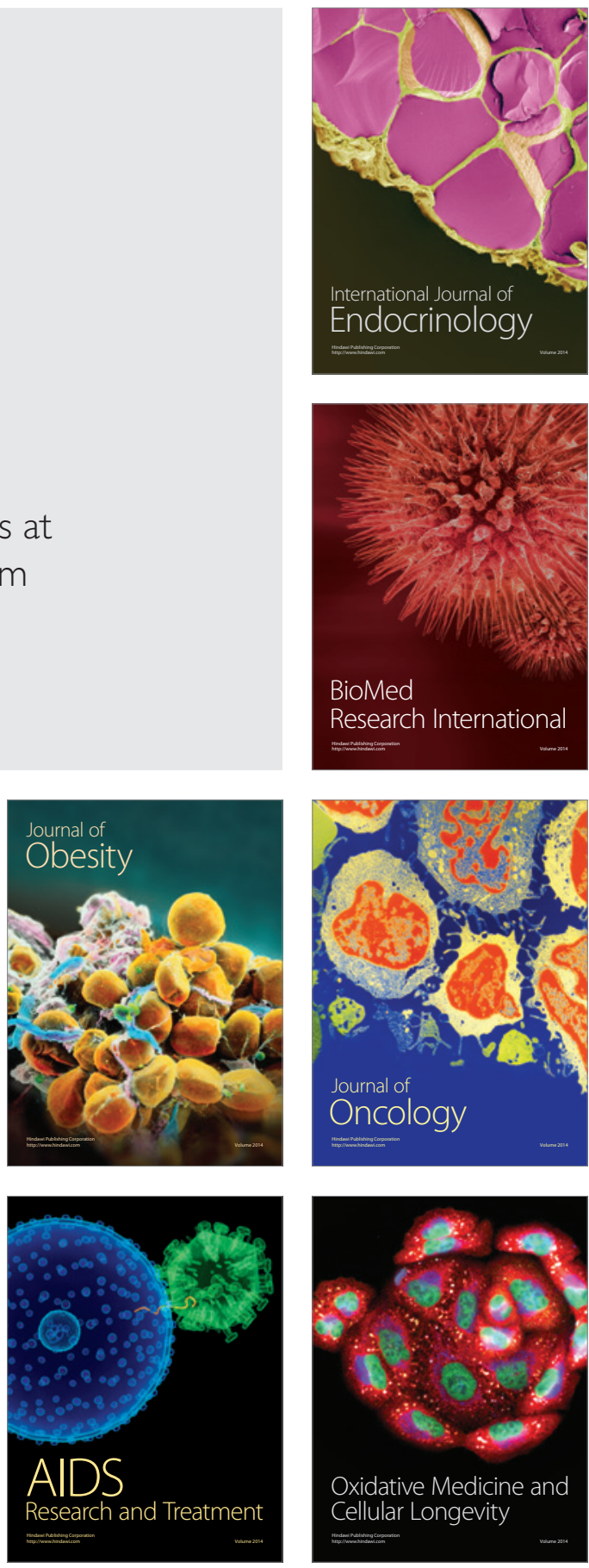